\title{
Die Konservierung der Töne. Ein Archiv für gesprochene Sprache
}

Im Rahmen einer Publikation, die vornehmlich Archiven und Sammlungen literarischer Texte oder bildkünstlerischer Werke gewidmet ist, mag die Präsentation eines Archivs für gesprochene Sprache vielleicht peripher oder gar exotisch wirken. Die Besonderheiten eines solchen Archivs ergeben sich nicht nur aus den spezifischen Kategorien von Medialität und Materialität, sondern auch aufgrund praktischer Fragen wie der Handhabung von Primär- und Metadaten, der Transkription und Annotation von Gesprächsdaten oder der archivtauglichen Langzeitsicherung. Da allerdings der Anteil digitaler Medien in allen Arten von Archiven ständig zunimmt, werden kategorielle Unterschiede zwischen den klassischen Literatur- und Kunstarchiven und den Ton- und Filmarchiven zunehmend geringer werden.

Als bescheidenes Beispiel eines Audio-Archivs soll im Folgenden das OÖ. Spracharchiv vorgestellt werden, das im Adalbert-Stifter-Institut des Landes Oberösterreich angesiedelt ist und neben schriftlichen Quellen zu den Dialekten des Landes vor allem Tondokumente beherbergt. Geführt und bestückt wird das Archiv von der hausinternen Einrichtung OÖ. Sprachforschung, die sich der Erforschung, Dokumentation und Beschreibung oberösterreichischer Sprachvarietäten in ihren vielfältigen regionalen, sozialen und medialen Erscheinungsformen widmet.

Das OÖ. Spracharchiv kann es hinsichtlich Alter und Bedeutung, Umfang und Erschließungsgrad natürlich keineswegs mit anderen traditionsreichen Spracharchiven - etwa dem „Archiv für gesprochenes Deutsch“ in Mannheim (vgl. Schmidt 2017) - aufnehmen: Es ist erst rund 25 Jahre alt, sein Sammelschwerpunkt sind die regionalen Sprachvarietäten eines Bundeslandes, die inhaltliche Erschließung ist bislang nur ansatzweise erfolgt, eine (Online-)Publikation der Tondokumente liegt noch in weiter Ferne. Von seiner Genese her ist das OÖ. Spracharchiv auch nicht das Ergebnis einer systematischen Sammeltätigkeit oder eines zielgerichteten Konzepts, sondern gleichsam nur Nebenprodukt des größeren Projektes Sprachatlas von Oberösterreich (SAO), bei dem neben den schriftlichen Aufzeichnungen an jedem Erhebungsort zusätzlich Tonaufnahmen eines oder mehrerer freier Gespräche gemacht wurden. Mit den Audio-Kassetten, Tonbändern und DAT-Kassetten, die pro Gemeinde etwa zwischen 30 und 60 Minuten freie Erzählungen enthalten, wurde im Jahr 1995 ein kleines AudioArchiv eingerichtet. Seither wurden die Materialien kontinuierlich vermehrt, sodass der Bestand an eigenen Tondokumenten gegenwärtig immerhin rund 
1.200 Stunden umfasst. Dazu kamen nach und nach auch digitalisierte Kopien historischer und aktueller Aufnahmen von Projektpartnern (insgesamt rund 210 Stunden), unter anderem frühe Phonogramme und Tonbänder aus dem Bestand des Phonogrammarchivs der Österreichischen Akademie der Wissenschaften oder Tonaufnahmen benachbarter Sprachatlasunternehmen, die dem OÖ. Spracharchiv zu Forschungszwecken überlassen wurden.

Wenn die historischen Aufnahmen von Projektpartnern hinzugenommen werden, kann behauptet werden, dass sich im Bestand des OÖ. Spracharchivs sowohl die wesentlichen technischen Entwicklungen als auch die diversen Paradigmen der Wissenschaftsgeschichte und deren Wechsel im Lauf des 20. Jahrhunderts spiegeln. Beide - die technischen und paradigmatischen Voraussetzungen lassen sich vielleicht am besten veranschaulichen, wenn wir uns entlang der Zeitleiste vom Beginn der Tonaufzeichnung bis zu gegenwärtigen Projekten bewegen.

\section{Beginn der Tonaufzeichnung: Phonogramme}

Die Anfänge der Phonographie, also der Aufzeichnung und anschließenden Wiedergabe von Schallereignissen, liegen im letzten Viertel des 19. Jahrhunderts. Als vor 120 Jahren auf Anregung des Physiologen Siegmund Exner-Ewarten das Phonogrammarchiv an der Kaiserlichen Akademie der Wissenschaften zu Wien eingerichtet wurde, wurden viele, auch geisteswissenschaftliche Disziplinen unter naturwissenschaftlichen Vorzeichen betrieben. So auch die Sprachwissenschaft, die nach den Erfolgen der Junggrammatiker bei der Rekonstruktion einer indoeuropäischen Sprachtypologie für die betreffenden Einzelsprachen spezifische und übergreifende Lautgesetze postulierte, die nach dem Vorbild der Naturgesetze ausnahmslos gelten sollten (vgl. Putschke 1969). Eine der wesentlichsten Subdisziplinen war die Phonetik, die damals vor allem als Lautphysiologie betrieben wurde. Ein wichtiges Hilfsmittel war dabei der Palatograph, eine technische Apparatur, mit der man die Stellungen der Zunge bei der Bildung der einzelnen Laute vermessen konnte. Die Entwicklung des Phonographen war ebenfalls ein wichtiger Baustein in diesem Programm. In Wien kam dabei nicht der von Thomas A. Edison patentierte Walzen-Phonograph zur Anwendung, sondern ein eigens entwickeltes Gerät, bei dem runde Wachsplatten als Tonträger dienten, auf die Schallereignisse mittels einer Nadel in „Tiefenschrift“ eingebracht wurden (vgl. Lechleitner 2018). Diese Platten hatten den Vorteil, dass sich von ihnen auf relativ einfache Weise Matrizen aus Metall herstellen ließen, die um vieles belastbarer als ihre Originale waren. Die Aufzeichnungs- und Spieldauer eines solchen Phonogramms betrug übrigens maximal 21/2 Minuten. 
$\mathrm{Zu}$ den ersten Phonogrammen, die in Wien angefertigt wurden, zählen sogenannte Stimmporträts bekannter Persönlichkeiten wie Kaiser Franz Joseph, Marie von Ebner-Eschenbach, Arthur Schnitzler, Joseph Weinheber und Albert Einstein. Die in der Kaiservilla in Ischl 1903 aufgenommene Stimme des Monarchen findet sich auf den Phonogrammen Nr. 1-3. Am Ende des von Exner vorgefertigten programmatischen Textes fügt der Kaiser noch die gewissermaßen spontanen Worte an: „Es hat mich sehr gefreut, auf Wunsch der Akademie der Wissenschaften meine Stimme in den Apparat hineinzusprechen und dieselbe dadurch der Sammlung einzuverleiben“ (Schüller 1997). Weitere thematische Schwerpunkte der Phonographie waren musikethnologische Studien auf verschiedenen Kontinenten (u. a. durch Rudolf Pöch in Neuguinea und in der Kalahari) sowie die Dokumentation von Sprachen ( $u$. a. durch Rudolf Trebitsch bei keltischen Minoritäten und bei den Basken). Darüber hinaus war es eine der Hauptaufgaben des Phonogrammarchivs, österreichische Mundarten im gesamten Gebiet der Habsburgermonarchie zu dokumentieren. Als älteste wissenschaftliche Dialektaufnahme im deutschsprachigen Raum gilt das Phonogramm Nr. 105, das die Schilderung einer Hirschjagd in Unterach am Attersee enthält (vgl. „Dazähl'n“ 2003, ID 5). In einer Reihe weiterer Phonogramme aus den Jahren 1901 bis 1909 wurden die sogenannten 40 Wenkersätze im jeweiligen Ortsdialekt aufgenommen. Dieser Fragenkatalog mit 40 einigermaßen konstruiert wirkenden Sätzen geht auf den Rheinländer Georg Wenker zurück, der um 1875 die Idee hatte, Fragebögen an alle Schulen in Norddeutschland, dann im Deutschen Reich und schließlich im gesamten deutschen Sprachraum zu versenden und die vorgegebenen Sätze von den Lehrkräften - und, wenn möglich, unter Mithilfe der Schüler - in die jeweilige Ortsmundart übersetzen zu lassen. Die Ergebnisse aus rund 50.000 Schulorten wurden von Wenker und seinen Nachfolgern im Sprachatlas des Deutschen Reichs bzw. im Deutschen Sprachatlas (DSA) ausgewertet (vgl. Schmidt und Herrgen 2011, 97-107). Wissenschaftsgeschichtlich gesehen liegt diesem dialektgeografischen Grundlagenwerk gewissermaßen ein erster „spatial turn“ zugrunde, der mit dem Dogma der Lautgesetze bricht oder zumindest deren Ausnahmslosigkeit bestreitet.

\section{Tonaufnahmen der Zwischenkriegs- und der NS-Zeit}

Nach dem Ersten Weltkrieg gerieten die Wiener und die Marburger dialektologische Schule im wissenschaftlichen Disput ziemlich heftig aneinander, wobei die Wiener Schule an den junggrammatischen Prinzipien und an der strengen phonetischen Ausrichtung festhielten (vgl. Wiesinger 1976). In den 1930er-Jahren zeigt die Sprachwissenschaft an der Wiener Germanistik einige fortschrittliche 


\section{Vordruckblatt.}

Sehulert:

Fragen.

1. Geselah die thersetzang durch den Lehrer oder dureh eine Mittelsperson?

2. Alter und Geschlecht des Ôbersetzers:


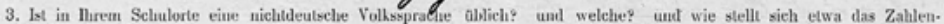
verhalltnis zwischen den von Haus aus Deutsehsprechenden und den Nicht-Deutschsprechenden?

4. Haben die Einwohner lhres Schulorts noch eine ausgeprägte Volkstracht? (Kurze Beschreibung wäre erwünscht.)
a) die Mănner?


b) die Franen:

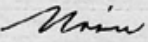

5. Wie lautet der Manute bes Sdjuforts in dortiger Mundart? und zwar

a) alleinstehend $=$

b) in dem Salze: $\boldsymbol{c} x$ wohut in ...



6. Wie lauten in Throm Sehulorte folgende Wörter*



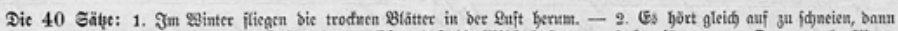

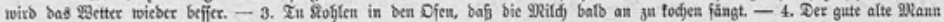

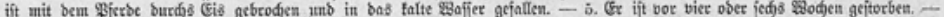

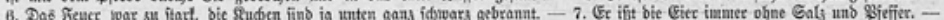

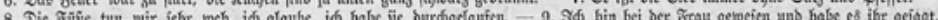

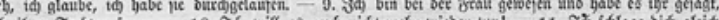

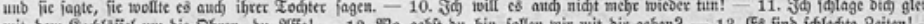



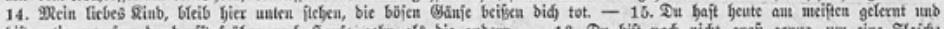

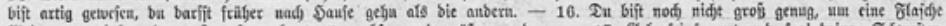

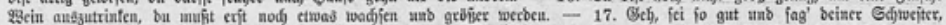



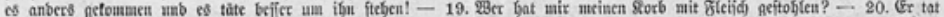

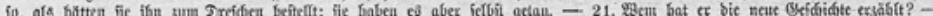
10. 22. 20.

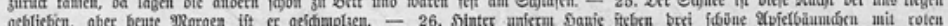

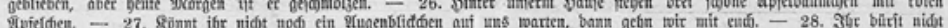



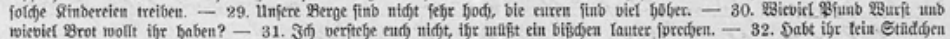

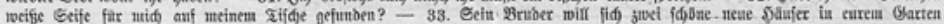

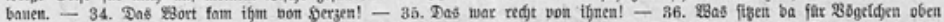

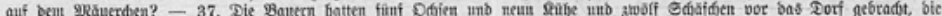

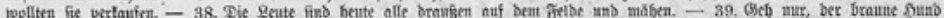

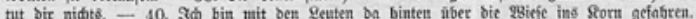

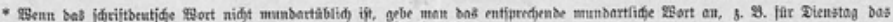



Abb. 1: Vorderseite eines Wenkerbogens aus der Gemeinde Hohenzell. 
Ansätze, indem sich vor allem der Sprachwissenschaftler Anton Pfalz früh dem Prager Strukturalismus öffnete und die phonologischen Anschauungen seines Kollegen Nikolai Trubetzkoy übernahm. Von dieser frühen Rezeption des Strukturalismus abgesehen, zeichnet sich die germanistische Sprachwissenschaft in Wien in den folgenden Jahrzehnten aber vor allem dadurch aus, dass sie nahezu alle wichtigen Strömungen der internationalen Linguistik des 20. Jahrhunderts negiert bzw. ignoriert hat.

In diesem Zusammenhang ist auch anzumerken, dass fast alle Vertreter des älteren Fachs und der Sprachwissenschaft einer deutschnationalen Gesinnung nachhingen und sich schließlich dem nationalsozialistischen Gedankengut zuwandten. Dies gilt insbesondere für Rudolf Much (1862-1936), Dietrich Kralik (1884-1959) und Anton Pfalz (1885-1958), die bereits in den 1920er-Jahren dem antisemitischen Professorennetzwerk „Bärenhöhle“ angehörten (vgl. Taschwer 2016). Im Fachbereich Sprachwissenschaft ist neben Pfalz auch noch Walter Steinhauser (1885-1980) zu nennen, der bereits 1932 der NSDAP beigetreten war, nach Kriegsende aus dem Universitätsdienst entlassen und 1949 als „Minderbelasteter“ in den Ruhestand versetzt wurde (vgl. Ranzmaier 2005, 45; König 2003, 1804). Pfalz und Steinhauser waren ab 1912 als Assistenten an der „Kanzlei zur Schaffung eines Bayerisch-Österreichischen Wörterbuches“ tätig, deren Leitung Pfalz von 1920 bis 1945 innehatte. Pfalz engagierte sich ab 1933 im NS-Lehrerbund, trat 1937 der NSDAP bei und wurde 1943 Pressereferent des NS-Dozentenbundes für die philosophische Fakultät der Universität Wien. 1945 aus dem Dienst entlassen, wurde auch er zwei Jahre später als „Minderbelasteter“ eingestuft und 1949 pensioniert (vgl. König 2003, 1396-1397). Schließlich ist auch noch der Dialektologe und Namenforscher Eberhard Kranzmayer (1897-1975) zu nennen, der nach wechselnder Tätigkeit in der Wiener und Münchner Wörterbuchkanzlei im Jahr 1938 zum Leiter der Bayerischen Wörterbuchkommission berufen wurde. Kranzmayer hatte bereits 1937 einen Antrag auf Mitgliedschaft in der NSDAP gestellt, wurde 1940 in die Partei aufgenommen und 1942 zum Leiter des „Instituts für Kärntner Landesforschung “ als Teil des SS-Ahnenerbes in Klagenfurt bestellt. Auch Kranzmayer erhielt nach Kriegsende Berufsverbot, das aber 1949 wieder aufgehoben wurde. Nach einer neuerlichen Anstellung bei der österreichischen Wörterbuchkommission erhielt er 1958 den Lehrstuhl für Deutsche Sprachwissenschaft und übernahm 1964 die Leitung der Wiener Wörterbuchkanzlei (vgl. Baur und Gradwohl-Schlacher 2011, 155-157).

Die hier genannten Details zu den Biografien der maßgeblichen Vertreter der Wiener germanistischen Sprachwissenschaft werden vor allem im Hinblick auf die wissenschaftliche Neuentdeckung einer Sammlung erwähnt, die 1936 vom „Reichsbund der deutschen Beamten“ initiiert wurde und im April 1937 dem Führer und Reichskanzler Adolf Hitler zu seinem 48. Geburtstag überreicht 
werden sollte. Es handelt sich dabei um das sogenannte Lautdenkmal reichsdeutscher Mundarten, das ursprünglich 300 Hörbeispiele deutscher Dialekte umfassen und damit einen repräsentativen Querschnitt durch alle Landschaften des Deutschen Reichs darstellen sollte (vgl. Wilking 2003, 203-220). Die wissenschaftliche Konzeption der Aufnahmen oblag den Marburger Sprachwissenschaftlern Bernhard Martin und Walther Mitzka, die technische Umsetzung erfolgte durch die Firma Telefunken, die acht Monate lang einen großen Aufnahmewagen durch das Land schickte. Von den Ortsaufnahmen wurden jeweils einzelne Schallplatten angefertigt, die in einem aufwändig gestalteten Holzschrank mit Landkarten und einem integrierten Abspielgerät aufbewahrt wurden. Die Überreichung dieses überdimensionalen „Dialekt-Wurlitzers“ an den Führer fand allerdings nicht an dessen Geburtstag, sondern erst verspätet am 30. Juni 1937 statt.

Unmittelbar nach dem „Anschluss“ Österreichs an das Deutsche Reich wurde über eine Erweiterung der Plattensammlung beraten. Die betreffenden Stellen in Wien waren offenbar schon länger darauf vorbereitet, denn bereits am 6. April 1938 kam es zur ersten Erhebungsfahrt in Hitlers Geburtsstadt Braunau. An den Aufnahmen beteiligt waren der deutsche Projektleiter Fritz Debus, die Wiener Professoren Anton Pfalz, Eberhard Kranzmayer und Dietrich Kralik sowie ein Techniker der Firma Telefunken. Österreichweit wurden 70 Orte für Sprachaufnahmen ausgewählt und nach der Eingliederung der Sudetengebiete noch weitere 30 Aufnahmen gemacht (vgl. Braun 2015). Allein schon die Auswahl der Erhebungsorte (Braunau, Leonding, Mauterndorf usw.) verweist auf den politischen Opportunismus dieses Unternehmens, umso mehr zeigt sich inhaltlich, dass viele Sprachaufnahmen vorbereitet und gelenkt waren und dass das Projekt insgesamt der Propaganda und der Gleichschaltung dienen sollte.

Nach dem Krieg hat die germanistische Fachwelt um die Aufnahmen des Lautdenkmals einen weiten Bogen gemacht. Über Walther Mitzka wird etwa berichtet, dass er noch während des Einmarschs US-amerikanischer Truppen seinen als Ehrengabe erhaltenen Plattensatz zerstört haben soll (vgl. Wilking 2003, 218). Erst Ende der 1990er-Jahre wurden die Platten in Deutschland gesichtet und digitalisiert. Die österreichischen Aufnahmen, die lange Zeit unbeachtet im Phonogrammarchiv lagerten, wurden erst 2014 vollständig erschlossen. Eine Besonderheit des österreichischen Korpusteils ist die Tatsache, dass neben den Aufnahmen auch die Kontextmaterialien (Aufnahmeprotokolle, Korrespondenzen usw.) erhalten geblieben sind (vgl. Braun 2015, 9-10). 


\section{Dialektaufnahmen der Nachkriegszeit}

Wie bereits angedeutet, hat die Wiener dialektologische Schule unter Eberhard Kranzmayer neuere Entwicklungen wie den Strukturalismus, die Psycho- und Soziolinguistik oder die generative Grammatik weitgehend ignoriert, weshalb es in der Wiener germanistischen Sprachwissenschaft kaum zu nennenswerten methodischen oder epistemologischen Fortschritten kam. Mit großer Intensität wurde jedoch die Erforschung mittelalterlicher Sprachinseln und konservativer Binnendialekte betrieben.

Mit der Einführung des Magnettonbands ab den 1950er-Jahren war es nunmehr bedeutend leichter, Feldforschungen mit Tonaufzeichnung durchzuführen. Zwischen 1951 und 1983 entstand so eine flächendeckende Audio-Dokumentation der österreichischen Dialekte, schon bis Ende 1952 wurden allein in Oberösterreich an die 70 Aufnahmen erbracht. Federführend bei diesen sogenannten Kundfahrten waren neben Kranzmayer dessen ehemalige Schülerin Maria Hornung, einige Mitarbeiter der Wiener Wörterbuchkanzlei (u. a. Albrecht Etz und Franz Roitinger) und ein Vertreter des Phonogrammarchivs (meist in Person von Walter Ruth). Themen der aufgenommenen Gespräche waren beispielsweise Haushalt und Landwirtschaft, das Almwesen, Feste und Unterhaltung, Brauchtum und Aberglauben (z. B. die Wilde Jagd), Sagen und Wilderergeschichten. Eines der Hauptmotive dieser regen Aufnahmetätigkeit war die akustische Dokumentation der besonders altertümlichen südbairischen Dialekte des alpinen Raums, die Kranzmayer in seiner romantischen sprachhistorischen Auffassung idealerweise in den Tiroler Hochtälern und in den im Mittelalter gegründeten zimbrischen Sprachinseln der Sieben und Dreizehn Gemeinden auf der Hochebene nördlich von Verona und Vicenza verkörpert sah. Im Bereich des tendenziell moderneren mittelbairischen Raums (zwischen Lech und Neusiedlersee mit den beiden Zentren München und Wien) waren für die Wiener Dialektologie vor allem die konservativen Mundarten Oberösterreichs von Interesse, insbesondere im Bereich der von Kranzmayer so genannten „oberösterreichischen Beharrsamkeitsbrücke“ (Kranzmayer 1956, 6). Im Herbst 2018 wurde dieses Korpus der „Tonaufnahmen österreichischer Dialekte 1951-1983“ in das nationale „Memory of the World“-Register der UNESCO aufgenommen. In Kooperation mit der Forschungsabteilung „Variation und Wandel des Deutschen in Österreich“ der Österreichischen Akademie der Wissenschaften sollen sie aufbereitet, erschlossen und künftig über eine Online-Plattform allgemein verfügbar gemacht werden (vgl. www.oeaw.ac.at/phonogrammarchiv/unesco). 


\section{Tonaufnahmen für den Sprachatlas von Oberösterreich (SAO)}

Wenn wir uns nun den Tondokumenten zuwenden, die auf eigene Initiative hin erstellt wurden, so sind zunächst die - bereits eingangs erwähnten - Aufnahmen freier Gespräche zu nennen, die im Kontext der schriftlichen Erhebungen des Sprachatlas von Oberösterreich (SAO) entstanden sind. Seit Beginn des Projekts im Jahr 1988 wurden pro Erhebungsort durchschnittlich zwei Aufnahmen zu je 30 Minuten gemacht, sofern nicht ein Defekt der altersschwachen Tonbandgeräte eine Aufzeichnung vereitelte. Themen dieser Interviews waren etwa bäuerliche Arbeiten, Feste, Bräuche, Sagen, Anekdoten, Jugenderinnerungen, Spiele, Musik, Kriegsereignisse usw. Die Form des ungesteuerten Interviews mit freier Themenwahl durch die GesprächspartnerInnen erbrachte in vielen Fällen originelle und auch aus der Sicht der Erzählforschung interessante Aufnahmen.

Einen regelrechten Quantensprung bildete - sowohl in qualitativer als auch in quantitativer Hinsicht - ab der Mitte der 1990er-Jahre der Einsatz digitaler Recorder und professioneller Großmembran-Mikrofone. Die DAT-Kassetten erlaubten bei einer Samplerate von $48 \mathrm{kHz}$ und einer nahezu rauschfreien Audioqualität zwei- bis dreimal längere Aufnahmen als die Tonbandspulen, außerdem haben sie sich in den letzten drei Jahrzehnten als relativ sichere Datenträger erwiesen.

Da die neue Technik die Speicherung großer Datenmengen auf kleinem Platz ermöglichte, wurde im Jahr 2001 damit begonnen, die regionalen Sprachvarietäten an allen SAO-Erhebungsorten systematisch $\mathrm{zu}$ dokumentieren. In einem Projekt mit dem Titel OöTon - Audiothek oberösterreichischer Dialekte wurden anhand eines Fragebogens 835 Fragen zu Wörtern, Phrasen und Sätzen mit phonologisch, morphologisch, syntaktisch und lexikalisch interessanten Phänomenen gestellt. Für die Erhebung des bäuerlichen Basisdialekts wurden Personen befragt, die der älteren Generation (Geburtsjahr vor 1950) angehören, einen landwirtschaftlichen Beruf ausüben bzw. ausgeübt haben und möglichst in der zweiten Generation ortsansässig sind. Aus dieser Enquete („OöTon - Serie I“) liegen bisher nahezu 540 Aufnahmestunden vor.

In einer weiteren Runde („Ö̈Ton - Serie II“) wurde der Versuch unternommen, den Sprachgebrauch einer jüngeren Altersgruppe zu erheben. Dafür wurden Personen befragt, die der Generation mit Geburtsjahr nach 1980 angehören und keinem landwirtschaftlichen Beruf nachgehen. Von dieser Serie liegen knapp 100 Aufnahmen vor, weitere 80 sind noch geplant. Die erhobenen Sprachdaten zeigen die Unterschiede im Sprachgebrauch zweier Altersgruppen auf und geben einen Einblick in die soziale Dimension sprachlicher Variation. $\mathrm{Zu}$ den Aufnahmen der OöTon-Enquete findet sich auf der Website des StifterHauses auch eine Anwendung, die Hörproben des traditionellen Dialekts und einer jeweils jüngeren Entsprechung bietet (vgl. https://stifterhaus.at/forschung/sprachforschung/hoeratlanten). Neben 
Tonbeispielen $\mathrm{zu}$ phonologischen, lexikalischen und morphosyntaktischen Variationsphänomenen ist auch ein Dialektquiz enthalten, das in zwei Schwierigkeitsgraden („Anfänger“, „Fortgeschrittene“) durchgespielt werden kann.

Mit dem jüngsten, erst Anfang 2016 in Angriff genommenen Projekt Sprachvariation in Oberösterreich (SVO) wird versucht, die gesamte Sprachvariation am Beispiel einer oberösterreichischen Gemeinde $\mathrm{zu}$ dokumentieren und die sozialen und situativen Faktoren der Sprachvariation zu erforschen. Dabei werden Frauen und Männer aus sechs Alters- und vier Berufsgruppen in unterschiedlichen kommunikativen Situationen beobachtet bzw. befragt und der jeweilige Sprachgebrauch dokumentiert. Für die Pilotstudie wurde die Gemeinde Weibern ausgewählt, wo versucht wird, das gesamte Variationsspektrum zwischen Dialekt und Standardsprache zu erfassen. Die Erhebung hat bislang mehr als 100 Stunden Tonmaterial erbracht.

\section{Deutsche Varietäten im östlichen Mitteleuropa}

Gleich nach der politischen Wende in Osteuropa hat sich Wilfried Schabus, damals Linguist am Phonogrammarchiv, nach Rumänien aufgemacht, um für den Sprachatlas von Oberösterreich Erhebungen bei den siebenbürgischen Landlern durchzuführen. Die Vorfahren dieser Minderheitengruppe, die vorwiegend aus dem Salzkammergut, dem oberösterreichischen Hausruckviertel und dem südlichen Kärnten kamen, waren ab dem zweiten Drittel des 18. Jahrhunderts aus politisch-religiösen Gründen in die Umgebung von Hermannstadt/Sibiu vertrieben worden. Weitere Aufnahmen führten Schabus zu SprecherInnen in der Karpaten-Ukraine, deren Vorfahren ebenfalls im 18. Jahrhundert, in diesem Fall aber aus vorwiegend wirtschaftlichen Gründen, in das damals ungarische Komitat Marmarosch ausgewandert sind, sowie zu Personen, die nach dem Krieg nach Sibirien deportiert worden waren.

Seit etwas mehr als zehn Jahren werden auch seitens des Adalbert-StifterInstituts Feldforschungen im östlichen Europa durchgeführt. Ein Schwerpunkt liegt dabei auf dem Banater Bergland im Bezirk Caraş-Severin südlich von Temeswar, wo neben der rumänischen Mehrheitsbevölkerung gleich mehrere sprachliche Minderheiten vorzufinden sind, die nicht selten auch innerfamiliär intensiveren Sprachkontakt pflegen (u. a. mit Rumänisch, Deutsch, Serbisch, Ungarisch, Kroatisch, Ukrainisch, Tschechisch, Slowakisch, Bulgarisch). Weitere geografische Schwerpunkte dieser Forschungen liegen in der Vojvodina im nordöstlichen Serbien sowie in Transkarpatien im Südwesten der Ukraine, wo ähnlich stark diversifizierte multiethnische und mehrsprachige Gesellschaften zu Hause sind. Die Aufnahmeserie firmiert unter dem Titel „Deutsch im östlichen Mitteleuropa“ 




Abb. 2: Interview mit einem ehemaligen Kapitän der DDSG-Agentie in Orschowa/Orşova, Rumänien.

(DoM), ihr Umfang beträgt bislang rund 150 Stunden. Auch zu den Ergebnissen dieser kontaktlinguistischen Forschungen sind Hörproben auf den Internetseiten des Adalbert-Stifter-Instituts zu finden (https://stifterhaus.at/forschung/sprachforschung/hoeratlanten).

\section{Ausblick}

Abschließend sei noch ein wichtiger Themenkomplex angesprochen, mit dem wohl alle konfrontiert sind, die mit analogen und/oder digitalen Medien zu tun haben. Dabei geht es unter anderem um folgende Fragen:

- die Handhabung und Verwaltung von Primär- und Metadaten

- die Digitalisierung analoger Medien

- die Transkription und Annotation von Audiodaten

- die langfristige Archivierung und Verfügbarkeit

- die eindeutige Referenzierbarkeit

- die Verwendung von Werkzeugen und Infrastruktur für den freien Gebrauch in der Forschung

- die Fragen des Urheberrechts und des Datenschutzes

Die hier genannten Fragen und Aufgaben konnten im OÖ. Spracharchiv bislang nur zum Teil gelöst werden. Weitgehend abgeschlossen ist die Digitalisierung analoger Medien, die teils im Phonogrammarchiv, teils im eigenen Institut erfolgt ist. Dazu gehören Tonbänder, die bereits nach 15-20 Jahren von Schichtablösun- 
gen betroffen waren und nicht mehr zur Gänze restauriert werden konnten, aber auch Sammlungen von Audio-Kassetten, die dem Archiv von Dritten überlassen wurden und wertvolles Material enthalten. $\mathrm{Zu}$ Letzteren zählen etwa Michael Kollmers Aufnahmen aus dem Bayerischen Wald (vgl. Kollmer 1987), die im OÖ. Spracharchiv digitalisiert und, mit Metadaten versehen, dem „Institut für deutsche Sprache“ (IDS) in Mannheim übergeben wurden, wo sie über die Datenbank für Gesprochenes Deutsch (DGD2) der Öffentlichkeit online zur Verfügung stehen.

Erst ansatzweise gelöst ist die Frage der Transkription, Annotation und Referenzierung der Audiodaten. Zwar wurde mit einer groben inhaltlichen Erschließung der Aufnahmen freier Gespräche begonnen, für eine systematische Transkription der Texte fehlen zurzeit jedoch die zeitlichen und finanziellen Ressourcen. Diese Schritte der inhaltlichen und formalen Erschießung bilden allerdings die Voraussetzung für eine mögliche (Online-)Publikation ausgewählter Bestände, die aus heutiger Sicht erst in ferner Zukunft realisierbar erscheint. Um ein Tonarchiv sinnvoll nutzen zu können, braucht es neben dem langfristig sichergestellten Zugriff auf die Tondokumente auch deren inhaltliche Erschließung sowie die technische Verknüpfung zwischen Audio- und Metadaten und den Transkriptionen.

Tonaufnahmen haben sicherlich einen besonderen, individuellen Charme. Das liegt möglicherweise auch daran, dass sie spezifische Informationen enthalten, die sich in schriftlichen Medien nur schwer vermitteln lassen. $\mathrm{Zu}$ diesen „suprasegmentalen“ Eigenschaften gehören unter anderem Phänomene wie Intonation, Modulation, Grundfrequenz und Obertöne, die selbst von differenzierten Transkriptionssystemen kaum adäquat erfasst werden können.

\section{Das 0ö Spracharchiv:}

\begin{tabular}{lr} 
Eigene Tonaufnahmen & \\
SAO - freie Gespräche (seit 1988) & $175 \mathrm{~h}$ \\
Fachsprachen (seit 2001) & $25 \mathrm{~h}$ \\
OöTon - Serie I (seit 2001) & $534 \mathrm{~h}$ \\
OöTon - Serie II (seit 2010) & $141 \mathrm{~h}$ \\
DoM (seit 2009) & $152 \mathrm{~h}$ \\
SVO - Pilotstudie Weibern (seit 2016) & $103 \mathrm{~h}$ \\
\hline gesamt & $\mathbf{1 . 1 3 0 ~ h}$ \\
& \\
Tonaufnahmen von Projektpartnern \\
Phonogramme (bis 1910) \\
Österreichische Dialekte (1951-1983) \\
Mondseeland (Dissertation Jakob Ebner) & $7,5 \mathrm{~h} \mathrm{~h}$ \\
Bayerischer Wald (Michael Kollmer) & $30,5 \mathrm{~h}$ \\
Sprachatlas von Niederbayern & $102 \mathrm{~h}$ \\
Bad Ischl (Diplomarbeit) & $21 \mathrm{~h}$ \\
Banat (West-Universität Temeswar) & $35,5 \mathrm{~h}$ \\
\hline gesamt & $\mathbf{2 0 7} \mathrm{h}$
\end{tabular}




\section{Literaturverzeichnis}

Baur, Uwe, und Karin Gradwohl-Schlacher. Literatur in Österreich 1938-1945. Handbuch eines literarischen Systems. Bd. 2: Kärnten. Wien, Köln, Weimar: Böhlau, 2011.

Braun, Jan. Das „Lautdenkmal reichsdeutscher Mundarten zur Zeit Adolf Hitlers“ in der "Ostmark“. Geisteswissenschaftliche Gemeinschaftsforschung am Beispiel der Germanistik von 1938 bis 1945. Wien: Univ. Masterarbeit, 2015.

Dazähl'n. 100 Jahre Dialektaufnahme in Österreich. Zusammengestellt und bearbeitet von Wilfred Schabus. Wien: Verlag der Österreichischen Akademie der Wissenschaften, 2003.

Kollmer, Michael. Die schöne Waldlersprach von Wegscheid bis Waldmünchen, von Passau bis Regensburg. 3 Bde. 1. Bd.: Lautliche und grammatische Beschreibung der Waldlersprache. 2. Bd.: Wortschatz und Redensarten der Waldlersprache. 3. Bd.: Waldler erzählen. Prackenbach, 1987.

König, Christoph. Internationales Germanistenlexikon 1800-1950. 3 Bde. Berlin, New York: de Gruyter, 2003.

Kranzmayer, Eberhard: Historische Lautgeographie des gesamtbairischen Dialektraumes. Wien, 1956.

Lechleitner, Gerda. „The Phonogrammarchiv of the Austrian Academy of Sciences“. Etnografie Sonore/Sound Ethnographies I/1. Hg. Giorgio Adamo, Sergio Bonanzinga und Nico Staiti. Roma: NeoClassica, 2018. 225-239.

Putschke, Wolfgang. „Zur forschungsgeschichtlichen Stellung der junggrammatischen Schule“. Zeitschrift für Dialektologie und Linguistik 36.1 (1969): 19-48.

Schmidt, Jürgen Erich, und Joachim Herrgen. Sprachdynamik. Eine Einführung in die moderne Regionalsprachenforschung. Berlin: Erich Schmidt, 2011.

Schmidt, Thomas. „DGD - die Datenbank für Gesprochenes Deutsch. Mündliche Korpora am Institut für Deutsche Sprache (IDS) in Mannheim“. Zeitschrift für germanistische Linguistik 45.3 (2017): 451-463.

Schüller, Dietrich (Hg.). Tondokumente aus dem Phonogrammarchiv der Österreichischen Akademie der Wissenschaften. Historische Stimmen aus Wien. Vol. 3: Kaiser Franz Joseph - Stimmporträt 1903. Mit einer Einführung von Helmut Rumpler. Wien, 1997.

Taschwer, Klaus. „Geheimsache Bärenhöhle. Wie ein antisemitisches Professorenkartell der Universität Wien nach 1918 jüdische und linke Forscherinnen und Forscher vertrieb“. Alma mater antisemitica: Akademisches Milieu, Juden und Antisemitismus an den Universitäten Europas zwischen 1918 und 1939. Bd. 3. Hg. Regina Fritz, Grzegorz Rossoliński-Liebe und Jana Starek. Wien: New Academic Press, 2016. 221-242.

Wiesinger, Peter. „Die Wiener dialektologische Schule“. Festgabe für Otto Höfler zum 75. Geburtstag. Hg. Helmut Birkhan. Wien: Braumüller, 1976. 661-693.

Wilking, Stefan. Der Deutsche Sprachatlas im Nationalsozialismus. Studien zu Dialektologie und Sprachwissenschaft zwischen 1933 und 1945. Hildesheim, Zürich, New York: Olms, 2003. 